\title{
Investigation on the effect of stimulated Raman scattering in remotely-pumped L-band erbium-doped fiber amplifier
}

\begin{abstract}
The effect of stimulated Raman scattering (SRS) in remote pumping scheme for L-band erbium-doped fiber amplifier (EDFA) was studied in this experiment. Observation was done on the gain and noise figure performance as well as the Raman distributed gains of the remotely-pumped L-band erbium-doped fiber amplifier with the existence of SRS. The utilization of SRS effect as a secondary pump for L-band EDFA demonstrated improvements over single pump EDFA at low pump power due to the additional power from the generated C-band stimulated Raman scattering or SRS. Nevertheless, the augmentation of the Cband SRS power as the pump was increased, caused gain competition with the L-band signal which ultimately affected the performance of the L-band EDFA. The Raman distributed gain was also influenced by the C-band SRS existence, which saw declining L-band gains as the C-band SRS rose and then improved values once the C-band SRS is saturated.
\end{abstract}

Keyword: Remotely-pumped optical amplifier; Stimulated Raman scattering; Erbium 